International Journal of Artificial Intelligence \& Applications (IJAIA), Vol.4, No.1, January 2013

\title{
AN INTEGRATED GENETIC-BASED MODEL OF NAIVE BAYES NETWORKS FOR CREDIT SCORING
}

\author{
Ali Zeinal Hamadani ${ }^{*}$, Ali shalbafzadeh ${ }^{2}$, Taghi Rezvan $^{3}$, and \\ AfshinShahlayi Moghadam ${ }^{4}$ \\ ${ }^{1}$ Department of Industrial Engineering, Isfahan University of Technology, \\ Isfahan 84156-83111, Iran. \\ *Corresponding author \\ hammadani@cc.iut.ac.ir \\ ${ }^{2}$ Department of Electrical and Computer Engineering, \\ Isfahan University of Technology, Isfahan, 84156-83111, Iran. \\ a.shalbafzadeh@ec.iut.ac.ir \\ ${ }^{3}$ Department of Industrial Engineering, \\ Isfahan University of Technology, Isfahan, 84156-83111, Iran. \\ taghi_rezvan@in.iut.ac.ir \\ ${ }^{4}$ Department of Industrial Engineering, \\ Isfahan University of Technology, Isfahan, 84156-83111, Iran. \\ afshin.shmegmail.com
}

\begin{abstract}
Inappropriate management in some fields such as credit allocation has imposed too many losses to financial institutions and even has forced some of them to go bankrupt. Moreover, large volume data sets collected by credit departments has necessitated utilizing highly accurate models with less complexities. Credit scoring models with classification and forecasting customers into two groups good and bad can dramatically reduce risks of granting credits to customers.

In this paper, a novel integrated approach for credit scoring problem is presented. This approach utilizes rough sets for feature selection during the data pre-processing phase and also adopts two hybrid sequences, Nä̈ve Bayes networks and genetic algorithm, to classify customers. In order to assess the competitive performance of the proposed approach, it has been executed on three credit scoring datasets from the University of California Irvine Machine Learning Repository. Computational results demonstrate that our approach has superior performance in terms of classification accuracy and achieves higher overall classification rate as compared to several other previous studies.
\end{abstract}

\section{Keywords}

Credit Scoring, Nä̈ve Bayes networks, Genetic algorithm, Rough sets theory. 
International Journal of Artificial Intelligence \& Applications (IJAIA), Vol.4, No.1, January 2013

\section{INTRODUCTION}

During recent years, inappropriate management in the US and Europe has imposed many losses to financial institutions such as banks and insurance companies and even has forced many of them to go bankrupt. On 1997, only in the United States, issuers of credit cards have reported 27.19 billion dollars of loss and these losses have increased to 31.91 billion dollars on 2006 [1].

One of the main duties of a financial institution is to develop some sets of models and techniques to enable them to predict bankruptcy and to assess credibility of customers [2, 3]. Credit scoring is based on the idea of segregation of customers of credit cards and applicants of granting loans into two sets of good and bad. Hence, this problem will be treated as a classification and forecasting problem [4]. However, some researchers have used clustering techniques for preprocessing input samples so that they can follow the credit scoring classification process more wise $[5,6]$.Statistical techniques and artificial intelligence are both used in credit scoring models. Logistic regression analysis (LRA) and linear Discriminant analysis (LDA) are two statistical techniques which are mostly used in credit scoring applications; however there have been some criticizes to such models since in this models it is assumed that the relation between dependent and independent variables is linear [7, 8]. By introduction of Quadratic Discriminant Analysis (QDA), researches prove that in comparison against LRA, QDA is more sensible to the model assumptions.

Because of the nature of the data sets scorings and inequality covariance matrices of the accepted and rejected sets, some researchers [9, 10] have criticized LRA and Thomas (2000)has reported that LRA and LDA are not accurate enough for credit scoring [11].

Since artificial neural networks can easily handle nonlinear relations among dependent and independent variables then they were the next choices for credit scoring and investigations reveal that their accuracy is much more than LRA and LDA [12, 13]. But long process of learning neural networks in finding topology of the optimal network has been a challenge for a long time. Also because of black-box feature of neural networks they do not have the ability of extracting the rules. Meanwhile, neural networks are reported to be more accurate in comparison against decision trees and the K- Nearest Neighbour [13].

Other artificial intelligence techniques such as evolutionary computations and genetic algorithms [14], support vector machines [15-18] have been reported to have more benefits than statistical methods and optimization models for assessing risks according to experimental results.

Combinatorial and hybrid models are based on statistical and AI tools and as example we can refer to neural discriminant models[19], neuro-fuzzy models [20, 21], hybrid model based on Bayesian approach for attribute selection and support vector machines for clustering [22], neural network models and support vector machines [15], fuzzy inference and decision trees [23], combinatorial artificial neural networks and Multivariate Adaptive Regression Splines (MARS) [24], hybrid models based on support vector machines and genetic algorithms [5], hybrid techniques based neighbourhood rough set and SVM [25] and multiple kernels multi-criteria programming approach based on evolution strategy (ES-MK-MCP) [26].

Credit scoring models mostly concentrate on the modelling and evaluation stages of the data mining process while data pre-processing is less considered. However this stage can have great impacts on improvement of the final model performance. Wang at el., have utilized rough sets 
International Journal of Artificial Intelligence \& Applications (IJAIA), Vol.4, No.1, January 2013

and tabu search for selecting the attributes of credit scoring models during the pre-processing stage [27]. With selecting attributes, accuracy of models such as logistics, radial basis functions, and support vector machines have not become worse.

Tsai and Wei investigated the performance of a single classifier as the baseline classifier to compare with multiple classifiers and diversified multiple classifiers by using neural networks based on three datasets [28].Nanni and Lumini investigated the performance of several systems based on ensemble of classifiers for bankruptcy prediction and credit scoring [29]. Xu et al. proposed hybrid approach using link analysis ranking techniques to pre-process samples into weighted information, and SVM techniques to build classifiers [30]. Li et al. introduced a linear combination of kernel functions to enhance the interpretability of credit scoring models, and propose an alternative to optimize the parameters based on the evolution strategy[31]. Wang et al. investigated the performance of three popular ensemble methods-Bagging, Boosting, and Stacking- based on four base learners, i.e., LRA, DT, ANN and SVM on credit scoring problem [32]. Chi and Hsu selected important variables by GA to combine the bank's internal behavioural scoring model with the external credit bureau scoring model to construct the dual scoring model for credit risk management of mortgage accounts [33].Capotorti and Barbanera suggested a hybrid model for classification based on the methodologies of rough sets, partial conditional probability assessments and fuzzy sets for classifying credit applicants into classes of risk on the basis of probability of default values [34].

In this paper, a novel integrated approach for credit scoring is presented which uses k-means for discretizing data sets and rough sets for selecting attributes and also utilizes two hybrid sequences, Naïve Bayes network and genetic algorithm, to classify customers into bad and good. This approach is executed and evaluated on three famous data sets from Germany, Australia, and Japan and then assessment metrics including accuracy, recall, precision, and F-measure are calculated and the performance of the developed models are compared.

This paper has some eminences including: first, it has adopted an integrated approach for modelling credit scoring which focuses on the data pre-processing stage and then tries to improve the performance of the credit scoring model by hybridizing the model using Naïve Bayes network and genetic algorithm. Second, the developed approach is tested and evaluated by three famous data sets and its better results are verified in comparison against other researches; third, implementation procedure of the rough set and genetic algorithm has led to lower computational complexities and as a result execution time has been decreased successfully. The last specification of the presented model is that by using genetic algorithm, capability of rule extraction from credit data sets has become possible. The last specification can be used for justifying rejected customers, conditional admission of a rejected customer, and constructing a paradigm for customers in order to make credits.

The rest of the paper is as follows. In section2, needed tools and algorithms including rough sets, genetic algorithm, and Naïve Bayes networks will be briefly reviewed. The developed integrated approach is presented in section 3 and computational results will be discussed in section 4 . Finally, conclusion remarks are discussed and future possible research subjects will be introduced in section 5 . 
International Journal of Artificial Intelligence \& Applications (IJAIA), Vol.4, No.1, January 2013

\section{THE TOOLS OF THE PROPOSED APPROACH}

The proposed integrated approach is based on three tools including: rough set in order to reduce the problem scale and Naïve Bayes network and genetic algorithm as classifiers. Here these tools and their related developed algorithms are briefly introduced.

\subsection{Rough Set}

Pawlak has introduced a rule based methodology using rough sets in order to handle problems with high level uncertainties and non-monotonous relations among attributes which makes statistical analysis of data a daunting task [35].

In rough sets, an information system like $S=<U, Q, V, f>$ is a reflection of a data set that describes the number of objects. In information system $S, U$ is a closed universe of $N$ objects $\left\{x_{1}, x_{2}, \ldots, x_{N}\right\}$ which is a non-empty finite set and $\mathrm{Q}$ is a non-empty finite set of $\mathrm{n}$ attributes $\left\{q_{1}, q_{2}, \ldots, q_{n}\right\}$ which demonstrates the objects. $V=U_{q \in Q} V_{q}$ where $V_{q}$ is the value of attribute $q$; $f: U \times Q \rightarrow V$ is an universal decision function where the information function for each $q \in Q$ and $x \in U$ is $f(x, q) \in V_{q}$.If in this information system $A \subseteq Q$ is subset of attributes and $x, y \in U$ are objects, then $x$ and $y$ are indiscernible if and only if for each $a \in A$, we have $f(x, a)=f(y, a)$.

In an information system $S$ a specific subset $A \subseteq Q$ of attributes determines an approximate space $A S(U, I N D(A))$ in $S$. For the sets $A \subseteq Q$ and $X \subseteq U$ a lower approximation of $A(A X)$

from the set $X$ in $A S$ and an upper approximation of $A(\bar{A} X)$ from the set $X$ in $A S$ are defined as relations (1):

$$
\begin{aligned}
& { }_{-}^{A} X=\left\{x \in U:[x]_{A} \cap X \neq \varnothing\right\}=\cup\left\{Y \in A^{*}: Y \subseteq X\right\} \\
& \bar{A} X=\left\{x \in U:[x]_{A} \cap X \neq \varnothing\right\}=\cup\left\{Y \in A^{*}: Y \cap X \neq \varnothing\right\}
\end{aligned}
$$

Boundary zone $A$ - from the set $X \subseteq U$ in $A S$ (uncertain zone from $\operatorname{IND}(A)$ ) is defined as below:

$B N_{A}(X)=\bar{A} X-A X$

To clarify the details of rough sets more, reader can refer to $[35,36]$

The below algorithm is recommended for rough sets:

Step1: Constructing dual difference structures among the objects.

Procedure of lowering the attributes: for each two objects $x_{\mathrm{i}}$ and $x_{\mathrm{j}}$ a vector $\mathrm{a}_{\mathrm{ij}}$ of the length $\mathrm{n}$ (number of attributes) is constructed according to Table 1 which demonstrates the difference of the two objects. 
International Journal of Artificial Intelligence \& Applications (IJAIA), Vol.4, No.1, January 2013

Table 1. Structure of the comparator of two records from the learning data set

\begin{tabular}{cccccccccc}
\hline$q_{0}$ & $q_{1}$ & $q_{2}$ & $q_{3}$ & $q_{4}$ & $q_{5}$ & $q_{6}$ & $q_{7}$ & $q_{8}$ & $q_{9}$ \\
\hline 1 & 0 & 1 & 1 & 0 & 0 & 1 & 1 & 0 & 0 \\
\hline
\end{tabular}

This vector demonstrates the difference between two objects as $q_{0} \wedge q_{2} \wedge q_{3} \wedge q_{6} \wedge q_{7}$. Output of this step is a list of polynomial structures similar to table 1.

Step2: Omission of similar polynomial structures.

In order to omit similar polynomial structures, we utilize an algorithm called tree K-means algorithm since it is similar to K-means and trees. The reason to use such an algorithm is its capability in omitting polynomial structures rapidly. Here this algorithm is described:

Algorithm tree K-means operates on the basis of two parameters K, number of clusters, and $\mathrm{F}$, maximum number of cluster members. Structures are being chosen from the beginning of the polynomial structures list of step 1 .

In the first level according to $\mathrm{K}$ some branches are established and a structure will be set in each branch. Then, according to Euclidean distance, $\mathrm{K}+1$ th structure is allocated to the closest cluster from $\mathrm{K}$. The allocation process lasts until the number of allocated structures to the cluster does not exceed F. The first structure to be allocated to each cluster will be the center of the cluster and will not be upgraded. When in a cluster there are F structures, if another structure wants to be added to it, then that cluster is divided in to $\mathrm{K}$ clusters of the lower level but the center of the upper level cluster will not be taken to the lower level. Hence, based on this methodology the whole structures in the list will be set in the tree or will be similar to the structure of clusters so that they will not be omitted.

Step 3: Merging the sentences

Polynomial structures are entirely arranged according to the number of factors one. Hence, a list is obtained where the merging procedure of its sentences having capability of merging will be as below:

3-1-Set the $i^{\text {th }}$ structure equal to zero and also set the $j^{\text {th }}$ structure equal to the last element of the list.

3-2-Compare the $i^{\text {th }}$ polynomial structure with the $j^{\text {th }}$ structure. If it is reducible then omit the $j^{\text {th }}$ polynomial structure and set the $i^{\text {th }}$ equal to the result of the difference.

A reducible polynomial structure is defined as follows. Two polynomial structures $\mathrm{x}$ and $\mathrm{y}$ can be reduced to $x$ if both of them have the parameter $q_{i}$ and also there is no parameter like $q_{i}$ in $x$ to be in $y$. Hence, this definition corresponds to $x \wedge \sim y=0$.

3-3-Reduce one unit from $j$

3-4- if $i<j$ then go to 3-2.

3-5- Add one unit to $i$.

3-6- If $I$ is not the last element, then go to 3-2. 
International Journal of Artificial Intelligence \& Applications (IJAIA), Vol.4, No.1, January 2013

Step 4: Determine reduction and core of the attribute.

Execute the step 2 on polynomial structures which cannot be merged anymore. The remaining sentences demonstrate the core and the reductions related to attributes.

\subsection{Naïve Bayes Networks}

In this section, basic concepts of Naïve Bayes networks in classifying problems are discussed. Such concepts can be further studied in [37].

Bayesian networks are directional acyclic graphs where their vertexes consist of information about the conditional probability values of a set of variables. In such networks, any sample x can be described only through the reference combination of its attributes such that these attributes are conditionally independent and the objective function $\mathrm{f}(\mathrm{x}): x \rightarrow v$ can have any value from the constrained set $v$. Any sample $x$ is displayed by vector $\left(a_{1}, \ldots, a_{n}\right)$ in which the most probable objective function value can be obtained by relation 3 . In this relation, $P\left(v_{j}\right)$ is calculated by counting the number of times that $v_{j}$ has been viewed in the training data set; but calculation of $P\left(a_{1}, \ldots, a_{n} \mid v_{j}\right)$ is rather impossible except in situations where the training data set is very large.

$v=\underset{v_{j} \in V}{\arg \max } P\left(a_{1}, \ldots, a_{n} \mid v_{j}\right) P\left(v_{j}\right)$

Using the easy assumption Naïve, in which values of attributes are conditionally independent, probability of viewing reference combination $\left(a_{1}, \ldots, a_{n}\right)$ for a determined objective function value, can be calculated by multiplying probability of each attribute. Therefore relation (4) is:

$v_{N B}=\underset{v_{j} \in V}{\arg \max } P\left(v_{j}\right) \prod_{i=1}^{n} P\left(a_{i} \mid v_{j}\right)$

Where probabilities $P\left(v_{j}\right)$ and $P\left(a_{i} \mid v_{j}\right)$ are estimated by the number of iterations. Totally these estimations construct an assumption that can be used in classifying new data. The algorithm incorporated in Naïve Bayes networks is the same existing algorithm which is widespread in researches [38-39].

\subsection{Genetic Algorithm}

Genetic algorithms are usually used in data mining for improving other algorithms or constructing association rules. Genetic algorithms operate on a population with different specifications in a determined framework. Genetic algorithms operational procedure is based on applying several operators including reproduction, crossover, and selection in a combination with mutation on genes of the initial population in order to generate an improved generation with better characteristics. For more details on applications of genetic algorithms in the field of data mining, reader is referred to [40-41]. 
International Journal of Artificial Intelligence \& Applications (IJAIA), Vol.4, No.1, January 2013

Structure of the designed gene for this problem consists of several chromosomes where each chromosome is an independent (conditional) attribute. This means that if a data set including $n$ attributes and one decision attribute, then each gene structure has $n+1$ chromosome. The structure of gene has been shown in Fig.1.

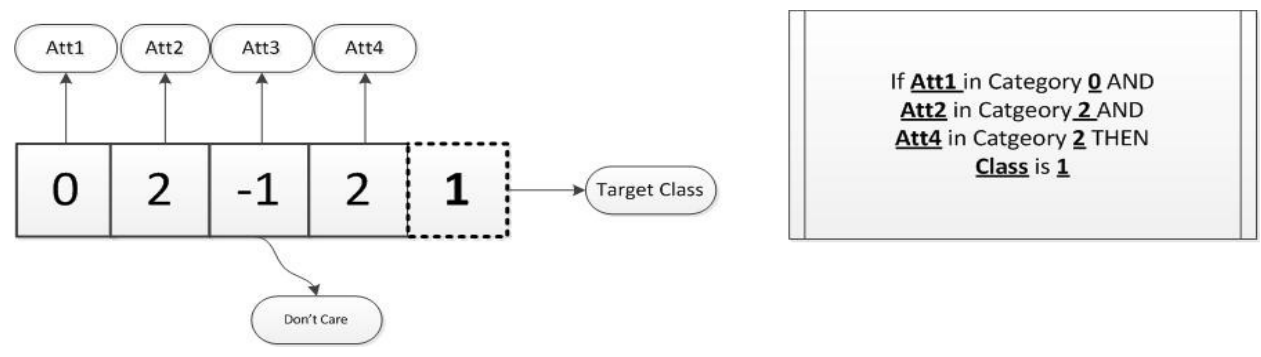

Figure 1. The structure of gene

For each gene a fitness function is defined according to equation (5). In this equation, $\beta$ is considered to be larger than $\alpha$. Fitness value is the gene score. Also sup port is the value that enables a gene, having conditional attributes, to cover the learning data set. Confidence is a value enables that a gene, with a non-zero sup port percent, to cover those decision attribute in a specific group.

Fitness $=\alpha^{*} \sup$ port $+\beta *$ confidence

In order to generate the initial population the value $N$ is taken from the objects of the learning data set and then some number of genes equal to the number of conditional attributes are generated and added to this population. The value of the chromosomes in the structure of these genes is entirely -1 except a remaining chromosome where allowed values of its corresponding attribute is randomly allocated to. Therefore, the initial values during the first iteration will be $N+$ "number of attributes".

Selection, mutation, and crossover operators operate according to predetermined probabilities $P_{\text {mutation }}$ and $P_{\text {crossover }}$ in such a way that during a mutation a chromosome is randomly selected and then a number is randomly selected from the set of possible values of the corresponding attributes of that chromosome and -1. But in crossover operation, a point is randomly. This point means a breaking point and crossover operator exchanges the two parts of the gene located on the both sides of this point.

Number of operations is considered as a percentage of $N$ and also in order to avoid computational complexities, the maximum of population in every iteration is considered to be a GA parameter than can be set. The number of operations and the maximum population are displayed by $\% \mathrm{Op}-\mathrm{Num}$ and $P O P_{\max }$ respectively. Also, if crossover operator performs on the population then it will just operate on a percentage of the population with high fitness that this parameter is showed by \%High Fitness. The below algorithm is recommended for GA: 
International Journal of Artificial Intelligence \& Applications (IJAIA), Vol.4, No.1, January 2013

Step1:Determining input parameters of the genetic algorithm $\left(\alpha, \beta, N, \% O p-N u m, P O P_{\max }\right.$, Iteration-Num, $P_{\text {mutation }}, P_{\text {crossover }}, \%$ High Fitness). The set parameters of the GA are determined by trial and errors which are summarized in Table2.

Step2: Generating the initial population

Step3: Calculating scores of each gene according to fitness function and arranging the population.

Step4: Operating mutation and crossover operators and adding the generated genes to the population

Step5: Until reaching Iteration-Num, execute steps 3 and 4.

During steps 3, 4, and 5 we should note that in each iteration, population size must not exceed the maximum population size set before. If this happens then remaining must be omitted from the arranged population.

Table2. Setting parameters of the genetic algorithm based on trial and errors.

\begin{tabular}{cc|cc}
\hline Parameter Name & Value & Parameter Name & Value \\
\hline$N$ & 200 & $P_{\text {crossover }}$ & $60 \%$ \\
$\% O p-N u m$ & 30 & $\%$ High Fitness & $30 \%$ \\
POP ${ }_{\max }$ & 300000 & $\alpha$ & 1 \\
Iteration-Num & 100 & $\beta$ & 4 \\
$P_{\text {mutation }}$ & $10 \%$ & & \\
\hline
\end{tabular}

During the last iteration, we reach an arranged population which is the set of extracted rules of the genetic algorithm. In order to increase the quality of the rules, two predetermined threshold levels for two "support" and "confidence" are being considered so that only high quality rules are kept.

\section{THE PROPOSED INTEGRATED APPROACH}

The proposed integrated approach is constructed based on the data mining process CRSP-DM. A scheme of the approach is depicted in Fig. 2. There are pseudo codes of the used tools for creating the proposed models. In this approach, during the preparation stage, discretization of data is done by K-means and attribute reduction is performed by rough set. Also combination of Naïve Bayes networks and genetic algorithm with two different sequences is utilized during the modeling stage. Hence, according to existence of two different states during the preparation stage, with and without attribute reduction, and two modeling states, four states are ultimately possible. Although Naïve Bayes networks and genetic algorithms can be considered individually during the modeling stage but it is proved that their accuracy is more in hybrid form so we individual states are not investigated here. Assessment of the developed models is performed by $\mathrm{K}$ - cross validation and also comparison of ordinary indexes.

Algorithm K-means which is used in discretization is to some extent different from the K-means algorithm used for clustering purposes. Second root of objects determines K. In order to allocate data to the determined sets we have: based on the number of sets, values of attributes are allocated to sets from the first of the line until each set has one value. Then, other values are allocated according to closeness to the center of the set. During each allocation, the center is being updated. This process continues until all the values are allocated. At the end, each set is being tagged and the allocated data to each set will poses the same tag. 


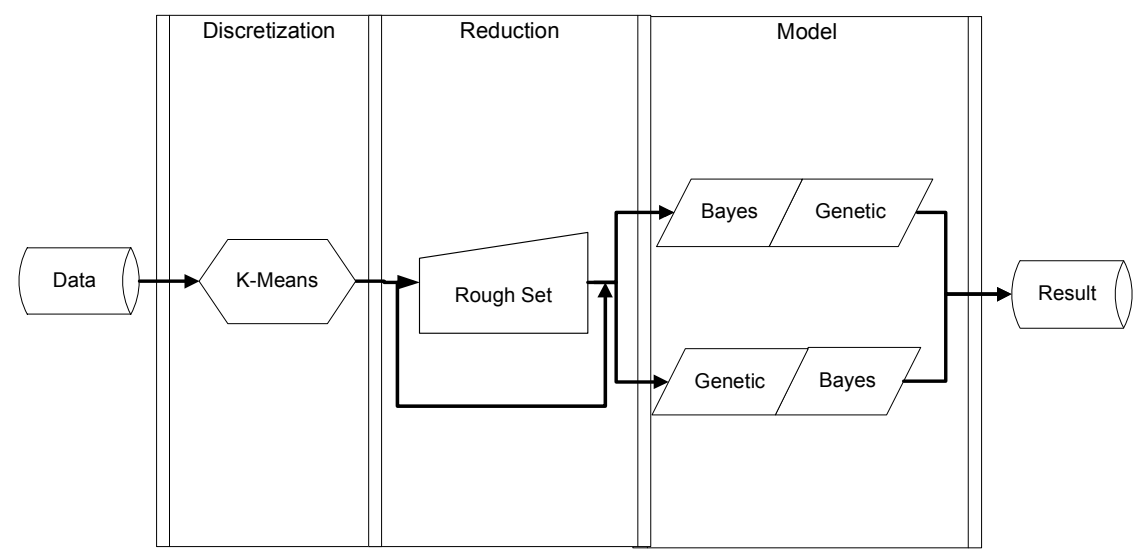

Figure 2. A schematic view of the proposed integrated approach

Since a probability is estimated for each class in Naïve Bayes networks then if the difference of estimated probabilities two classes in the hybrid model is less than the predetermined difference then the winner class is determined by the rules extracted by the genetic algorithm. In the hybrid model of the GA and Naïve Bayes networks, if the extracted rules by the GA are not able to determine the new object set then Naïve Bayes classifier will be used to determine the class.

The proposed integrated approach introduced in section 3 led to 4 models called "Not Rough +NB +GA", "Rough +NB+GA", "Not Rough+ GA+NB" and "Rough +GA+NB". Flowchart of models Rough $+\mathrm{NB}+\mathrm{GA}$ " and "Rough $+\mathrm{GA}+\mathrm{NB}$ " is shown in Fig. 3.In other two models, there is notthe reduction stage by rough set theory.

\section{EXPERIMENTAL ANALYSIS}

\subsection{Real world credit data sets}

Three real world data sets including Australian, German, and Japanese data sets are depicted in table3. These data sets can be accessed from UCI Repository of Machine Learning Databases which is adopted to assess the newly developed models [42]. The German data set is not balanced where 700 customer samples are good and 300 customer samples are bad. For each applicant, 20 conditional attributes are registered: history, account balances, loan purpose, loan amount, employment status, personal information, age, housing, and job title. Australian and Japanese data sets are nearly balanced and include 307 customers with good credit and 383 customers with bad credit and the difference between the registered attributes for each applicant in the two data sets is only in a single numerical attribute. In Japanese data set numerical and nominal miss value data are completed using mean and mode, respectively 
International Journal of Artificial Intelligence \& Applications (IJAIA), Vol.4, No.1, January 2013
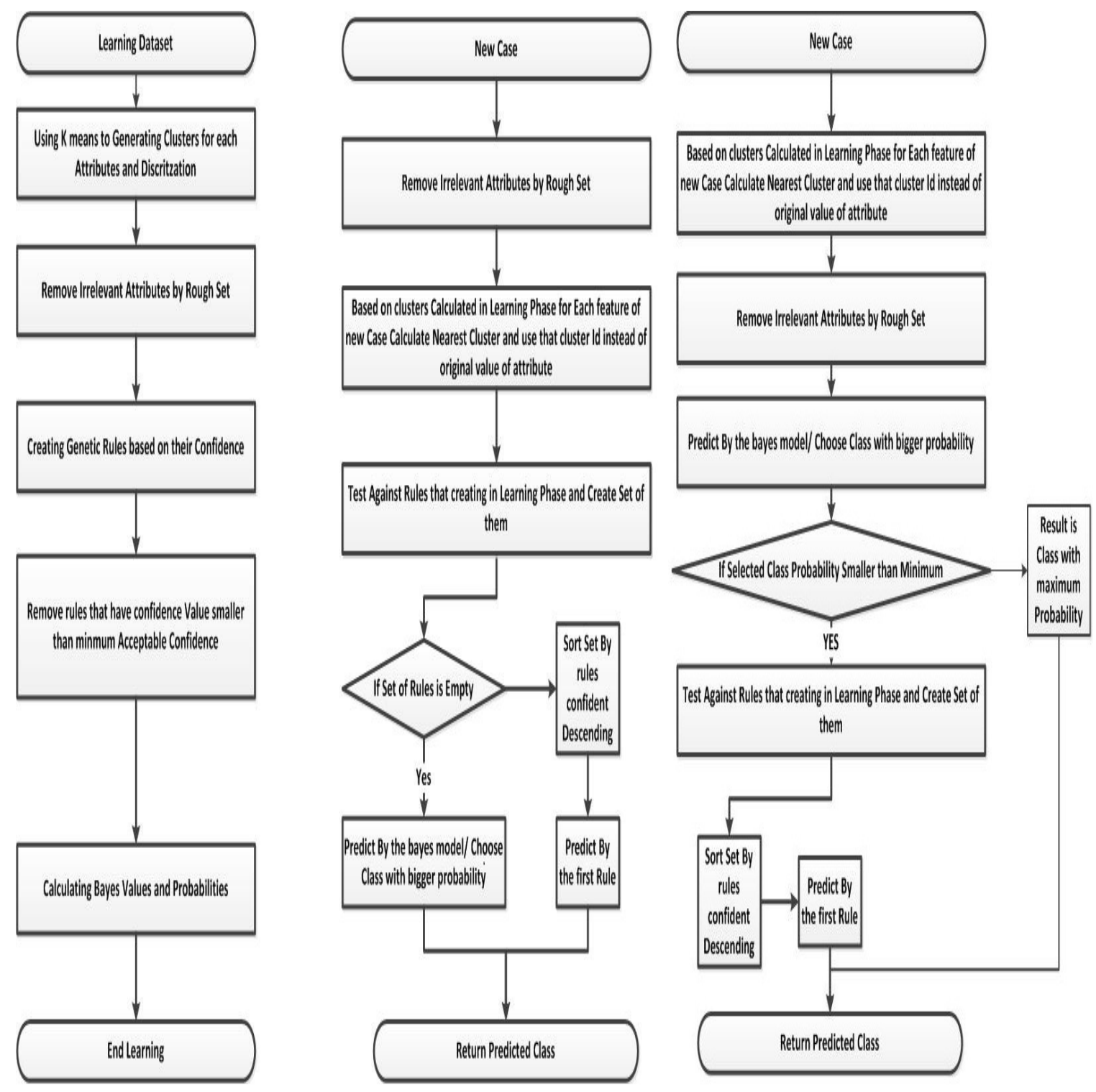

Figure 3. Learning process, "Rough $+\mathrm{GA}+\mathrm{NB}$ " and Rough $+\mathrm{NB}+\mathrm{GA}$ "

(respectively from left to right)

\subsection{Results of the proposed models}

The models "Not Rough +NB +GA", "Rough +NB+GA", "Not Rough+ GA+NB" and "Rough $+\mathrm{GA}+\mathrm{NB}$ " are executed on three data sets from Germany, Australia, and Japan and the computational results are summarized in tables 4,5 , and 6 . 
International Journal of Artificial Intelligence \& Applications (IJAIA), Vol.4, No.1, January 2013

Table 3. Credit scoring databases from the UCI Repository

\begin{tabular}{|c|c|c|c|c|c|}
\hline \multirow{2}{*}{ Names } & \multirow[t]{2}{*}{$\begin{array}{c}\# \\
\text { Instances }\end{array}$} & \multirow{2}{*}{$\begin{array}{c}\# \\
\text { Instances Of } \\
\text { Creditworthy } \\
\text { Class }\end{array}$} & \multicolumn{2}{|c|}{$\begin{array}{c}\text { Conditional } \\
\text { Features }\end{array}$} & \multirow[t]{2}{*}{$\begin{array}{c}\text { Total } \\
\text { Features }\end{array}$} \\
\hline & & & $\begin{array}{l}\text { Numeric } \\
\text { Feature }\end{array}$ & $\begin{array}{l}\text { Nominal } \\
\text { Feature }\end{array}$ & \\
\hline German & 1000 & 700 & 13 & 7 & 21 \\
\hline Australian & 690 & 307 & 8 & 6 & 15 \\
\hline Japanese & 690 & 307 & 9 & 6 & 16 \\
\hline
\end{tabular}

According to table4, classification accuracies for the German data set for the four models are $83.60 \%, 88.00 \%, 84.00 \%$, and $88.20 \%$, respectively. These values are obtained for models which use rough set for reducing the attributes of their data set. It can be seen that the sequence GA+NB is slightly better than NB+GA. Index values of Precision parameters in contrast to Recall is low which demonstrates that the developed models for prediction of creditworthy class are more accurate in comparison with the other class.

Table 4. Summarized results with 5-cross validation for German credit data set

\begin{tabular}{ccccccccc}
\hline \multirow{2}{*}{ Model Name } & \multicolumn{2}{c}{ Accuracy } & \multicolumn{2}{c}{ Recall } & \multicolumn{2}{c}{ Precision } & \multicolumn{2}{c}{ F-measure } \\
\cline { 2 - 9 } & $\begin{array}{c}\text { Avg. } \\
(\boldsymbol{\%})\end{array}$ & $\begin{array}{c}\text { Std. } \\
(\boldsymbol{\%})\end{array}$ & $\begin{array}{c}\text { Avg. } \\
(\boldsymbol{\%})\end{array}$ & $\begin{array}{c}\text { Std. } \\
(\boldsymbol{\%})\end{array}$ & $\begin{array}{c}\text { Avg. } \\
(\boldsymbol{\%})\end{array}$ & $\begin{array}{c}\text { Std. } \\
(\boldsymbol{\%})\end{array}$ & $\begin{array}{c}\text { Avg. } \\
(\boldsymbol{\%})\end{array}$ & $\begin{array}{c}\text { Std. } \\
(\boldsymbol{\%})\end{array}$ \\
\hline $\begin{array}{c}\text { "Not } \\
\text { Rough+NB+GA" }\end{array}$ & 83.60 & 1.06 & 93.51 & 2.61 & 84.73 & 3.06 & 88.84 & 1.24 \\
"Rough+NB+GA" & 88.00 & 2.15 & 96.57 & 1.37 & 87.57 & 2.81 & 91.83 & 1.54 \\
"Not Rough+ & 84.00 & 1.37 & 94.04 & 2.18 & 84.79 & 2.73 & 89.13 & 1.21 \\
$\begin{array}{c}\text { GA+NB" } \\
\text { "Rough+GA+NB" }\end{array}$ & 88.20 & 2.20 & 96.72 & 1.07 & 87.70 & 2.92 & 91.96 & 1.57 \\
\hline
\end{tabular}

According to table5, classification accuracies for the Australian data set for the four models are $88.41 \%, 92.61 \%, 85.65 \%$, and $88.12 \%$, respectively and accuracy of the models which have used rough set algorithm to reduce attributes are higher. Since the values of other evaluation indexes are high then one can say that the developed models have the same performance for prediction in the both classes.

Table 5. Summarized results with 5-cross validation for Australian credit data set

\begin{tabular}{ccccccccc}
\hline & \multicolumn{2}{c}{ Accuracy } & \multicolumn{2}{c}{ Recall } & \multicolumn{2}{c}{ Precision } & \multicolumn{2}{c}{ F-measure } \\
\cline { 2 - 9 } Model Name & $\begin{array}{c}\text { Avg. } \\
(\boldsymbol{\%})\end{array}$ & $\begin{array}{c}\text { Std. } \\
(\boldsymbol{\%})\end{array}$ & $\begin{array}{c}\text { Avg. } \\
(\boldsymbol{\%})\end{array}$ & $\begin{array}{c}\text { Std. } \\
(\boldsymbol{\%})\end{array}$ & $\begin{array}{c}\text { Avg. } \\
(\boldsymbol{\%})\end{array}$ & $\begin{array}{c}\text { Std. } \\
(\boldsymbol{\%})\end{array}$ & $\begin{array}{c}\text { Avg. } \\
(\boldsymbol{\%})\end{array}$ & $\begin{array}{c}\text { Std. } \\
(\boldsymbol{\%})\end{array}$ \\
\hline $\begin{array}{c}\text { "Not } \\
\text { Rough+NB+GA" }\end{array}$ & 88.41 & 3.12 & 87.95 & 1.72 & 86.40 & 4.40 & 87.10 & 3.02 \\
$\begin{array}{c}\text { Rough+NB+GA" } \\
\text { "Not Rough+ }\end{array}$ & 92.61 & 1.88 & 90.56 & 3.38 & 92.83 & 3.80 & 91.60 & 2.07 \\
$\begin{array}{c}\text { GA+NB" } \\
\text { "Rough+GA+NB" }\end{array}$ & 85.65 & 3.49 & 85.64 & 1.95 & 82.92 & 5.47 & 84.21 & 3.45 \\
\hline
\end{tabular}


International Journal of Artificial Intelligence \& Applications (IJAIA), Vol.4, No.1, January 2013

According to table6, classification accuracies for the Japanese data set for the four models are $92.12 \%, 85.91 \%, 92.42 \%$, and $86.67 \%$, respectively and accuracy of the models which have used rough set algorithm to reduce attributes are higher. Comparison of models 1 and 3 with models 2 and 4 shows the accuracy of the models without rough set to be meaningful. Also by comparing the accuracy values we can conclude that the sequence "GA+NB" is slightly better than "NB+GA". On the other hand, low value of the attribute Recall, especially in models 2 and 4, demonstrates that the prediction power of models for the Creditworthy class is weaker than the other class.

Table 6. Summarized results with 5-cross validation for Japanese credit data set

\begin{tabular}{|c|c|c|c|c|c|c|c|c|}
\hline \multirow[b]{2}{*}{$\begin{array}{l}\text { Model } \\
\text { name }\end{array}$} & \multicolumn{2}{|c|}{ Accuracy } & \multicolumn{2}{|c|}{ Recall } & \multicolumn{2}{|c|}{ Precision } & \multicolumn{2}{|c|}{ F-measure } \\
\hline & $\begin{array}{l}\text { Avg. } \\
(\%)\end{array}$ & $\begin{array}{l}\text { Std. } \\
(\%)\end{array}$ & $\begin{array}{l}\text { Avg. } \\
(\%)\end{array}$ & $\begin{array}{l}\text { Std. } \\
(\%)\end{array}$ & $\begin{array}{c}\text { Avg. } \\
(\%)\end{array}$ & $\begin{array}{l}\text { Std. } \\
(\%)\end{array}$ & $\begin{array}{l}\text { Avg. } \\
(\%)\end{array}$ & $\begin{array}{l}\text { Std. } \\
(\%)\end{array}$ \\
\hline "Not & 92.12 & 5.37 & 77.10 & 35.18 & 82.91 & 20.43 & 77.71 & 31.26 \\
\hline Rough+NB+GA" & & & & & & & & \\
\hline "Rough+NB+GA" & 85.91 & 5.35 & 68.43 & 14.45 & 80.43 & 30.16 & 73.19 & 22.45 \\
\hline $\begin{array}{c}\text { "Not Rough+ } \\
\text { GA+NB" }\end{array}$ & 92.42 & 5.19 & 78.07 & 35.83 & 83.04 & 20.42 & 78.26 & 31.52 \\
\hline "Rough+GA+NB" & 86.67 & 5.88 & 68.43 & 14.45 & 83.24 & 28.04 & 74.69 & 20.90 \\
\hline
\end{tabular}

Performance of the developed models on data sets from Germany, Australia, and Japan in ROC space are depicted in figure 4, 5, and 6, respectively. It is apparent that in this space, a point is better which has a higher true positive rate and lower false positive rate. Points in Fig. 4 show that the models "Rough+GA+NB" and "Rough+NB+GA" have a better performance in comparison against others for the German data set because they are located at the north-western corner of ROC space. Also according to Fig. 5 and Fig.6, it can be concluded that the models "Not Rough+GA+NB" for Australian data set and models "Not Rough+GA+NB" and "Not Rough+NB+GA" for the Japanese data set have the best performance.

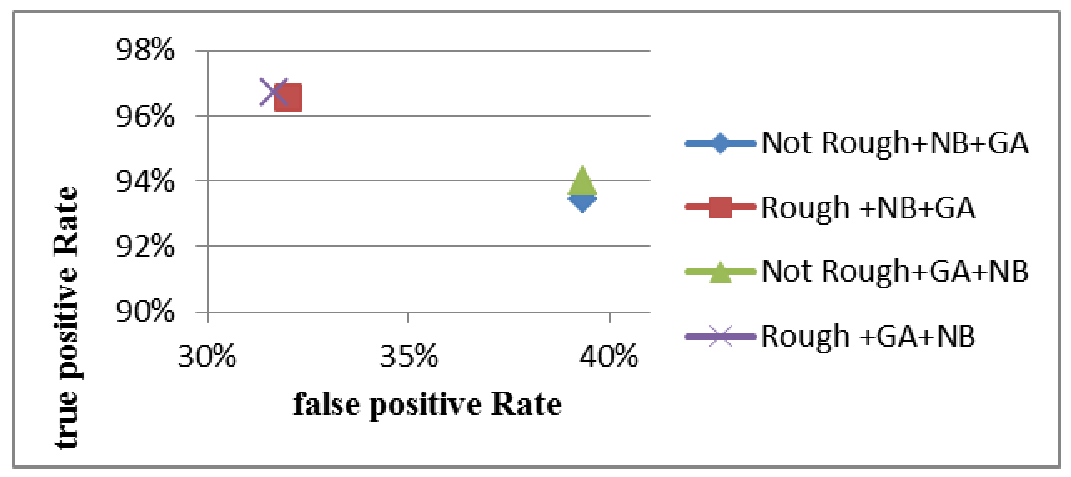

Figure 4. Comparison of model performances in ROC space for the German data set 
International Journal of Artificial Intelligence \& Applications (IJAIA), Vol.4, No.1, January 2013

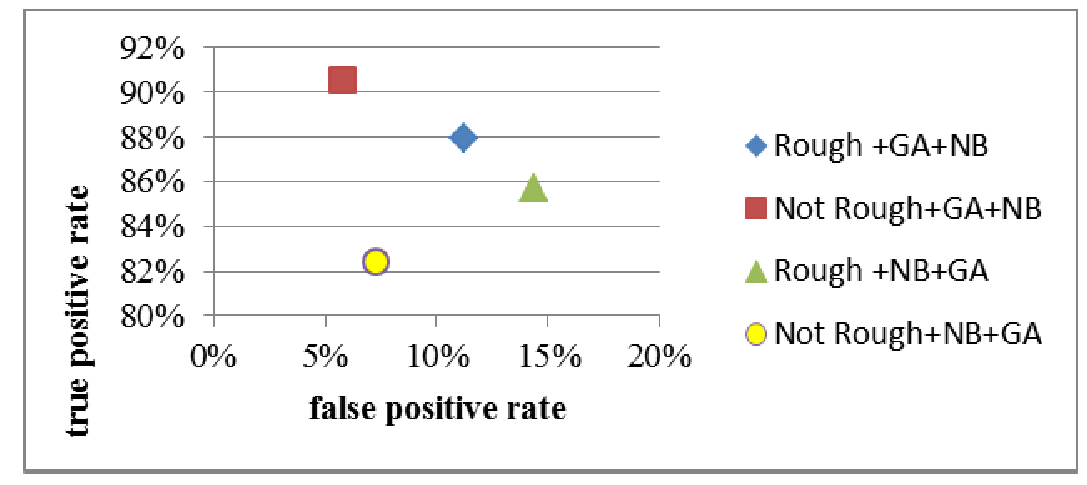

Figure 5. Comparison of model performances in ROC space for the Australian data set

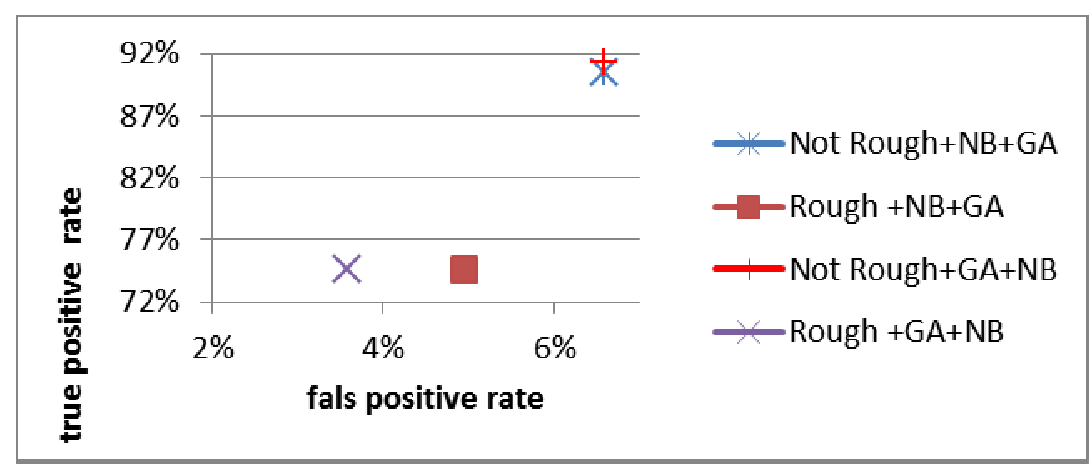

Figure 6. Comparison of model performances in ROC space for the Japanese data set

According to the obtained results from Tables and Figures it can be concluded that it is better to test rough set algorithm on such data sets which have more conditional attributes. On the other hand, it is not reasonable to conclude that if a model operates well on a data set then it will necessarily give the same result on another data set. In fact, this proves behavioral differences between customer who are applying for credits based on their culture and their socio-economic situation. As a conclusion, if someone wants to perform this process on another country, then other integrated models must be tested on its data set so that a more efficient model is obtained.

\subsection{Comparisons of different models}

In order to evaluate the effectiveness of the proposed credit scoring models, the obtained results are also compared with other approaches developed in the recent literature. It can be concluded, from Table 7, that the proposed classifier has the best credit scoring capability in terms of the overall classification rate. 
International Journal of Artificial Intelligence \& Applications (IJAIA), Vol.4, No.1, January 2013

Table 7. Accuracies with the different methods for Australian, German and Japanese data sets

\begin{tabular}{ccccc}
\hline \multirow{2}{*}{ Author (year) } & method used & \multicolumn{3}{c}{ Accuracy rate (\%) } \\
& Australian & German & Japanese \\
\hline Tsai and Wu (2008) [28] & neural network ensembles & 88.09 & 79.38 & 86.98 \\
& $\begin{array}{c}\text { clustering-launched } \\
\text { classification }\end{array}$ & 86.52 & 84.80 & - \\
Luo et al. (2009) [6] & GA+SVM & 86.9 & 77.92 & - \\
& Random Subspace ensemble & 87.05 & 73.93 & 87.34 \\
Nanni and Lumini (2009) & methods & & & \\
[29] & MLP & 85.74 & 75.00 & 86.96 \\
Ping and Yongheng & Neighborhood rough set and & 87.52 & 76.60 & - \\
(2011)[25] & SVM & & & \\
Wang et al. (2011) [32] & LRA & 86.56 & 76.14 & - \\
& DT & 84.39 & 72.10 & - \\
& ANN & 83.28 & 71.43 & - \\
Li et al. (2011) [26] & SVM & 85.67 & 76.28 & - \\
Jabeen and Baig (2012) [43] & ES-MK-MCP & 89.01 & 78.92 & - \\
& Two layered Genetic & 90.79 & 79.00 & - \\
\hline & Programming & & & \\
\hline & "Not Rough+NB+GA" & 88.41 & 83.60 & 92.12 \\
"Rough+NB+GA" & $\mathbf{9 2 . 6 1}$ & 88.00 & 85.91 \\
& "Not Rough+ GA+NB" & 85.65 & 84.00 & $\mathbf{9 2 . 4 2}$ \\
& "Rough+GA+NB" & 88.12 & $\mathbf{8 8 . 2 0}$ & 86.67 \\
\hline
\end{tabular}

\section{CONCLUSIONS AND FUTURE RESEARCHES}

Credit scoring is known to be one of the techniques used for reducing the risks of granting credits to customers of banks and financial institutions and is considered as a classification problem in the field of datamining. Presenting hybrid integrated models, this paper has increased efficacy of credit scoring models. Such an improvement is demonstrated by testing on three data sets from Germany, Australia, and Japan. According to the results, we can conclude that the improvements on performance of the models in this research in comparison against past studies are remarkable.The model "Not Rough+GA+NB" is also more efficient on the three tested data sets comparing to other models. Although power of models in predicting two classes of the three sets were different and prediction power of the model on the German data set in not-Creditworthy class was better but for Japan case situation of the other class is better. Also equal power of models for the both classes is verified by tests on the Australian model.

Developing a probabilistic or fuzzy model so that enables us to determine how much a customer belongs to good or bad groups can be reasonable. Development of credit scoring models for future researches can include models which assist customers in increasing his credit or in justifying him. In other words, customer finds out his credit weaknesses. 
International Journal of Artificial Intelligence \& Applications (IJAIA), Vol.4, No.1, January 2013

\section{Acknowledgement}

This paper is dedicated to the memory of Dr. Ali Arkan, friend and colleague, who passed away during submission of the manuscript.

\section{Appendix}

Pseudo code of the used tools for creating the proposed models are as follows:

\section{K-means for discretization}

///In learning phase

for $\mathrm{c}=1$ to Feature Count

For $\mathrm{n}=1$ to $\mathrm{K}(\mathrm{c})$

$\operatorname{Count}(\mathrm{c}, \mathrm{n})=0$

$\mathrm{m}(\mathrm{c}, \mathrm{n})=$ feature $(\mathrm{c})$ from (Random ItemSetin dataset)

For each ItemSet $\mathrm{s} 1$ in dataset

for each feature $f$ in Dataset

Select $\mathrm{n}$ such that EqlidianDistance[ $\mathrm{m}(\mathrm{f}, \mathrm{n}), \mathrm{s} 1(\mathrm{f})]$ is minimum

Count $(\mathrm{c}, \mathrm{n})+=1$

//In prediction phase

$$
\mathrm{m}(\mathrm{c}, \mathrm{n})=(\mathrm{s} 1(\mathrm{f})-\mathrm{m}(\mathrm{c}, \mathrm{n})) / \operatorname{Count}(\mathrm{c}, \mathrm{n})
$$

For each feature $\mathrm{f}$ in Dataset

Select $\mathrm{n}$ such that EqlidianDistance[ $\mathrm{m}(\mathrm{f}, \mathrm{n}), \mathrm{s} 1(\mathrm{f})]$ is minimum

$\mathrm{s} 1(\mathrm{f})=\mathrm{n}$

\section{Rough Set Theory for reduction}

Foreach pair of records row1, row2 in dataset

Generate Polynomial p(p1 or $\mathrm{p} 2$ or $\mathrm{p} 3$ or ..pn) where pi is column where row1.ci <>row2.ci

Add $\mathrm{p}$ to set(s)

Reducing Polynomial

Create List (L) from set(s)

Sort List (L) by length of polynomials

For each pair of Polynomial P1,P2 in List L

Check if $\mathrm{P} 1 \wedge$ ${ }^{\wedge} 2=\mathrm{P} 1$ then remove $\mathrm{P} 2$ from $\mathrm{L}$

//End of simplifying list of polynomials

While List (L) is not Empty

Select Polynomial P1, P2 from List(L) and remove them

Generate CNF form of (P1^P2) and add to List (L2)

Sort List(L2) by length of polynomials

For each pair of Polynomial P1, P2 in List L2

Check if $\mathrm{P} 1$ ? $\mathrm{P} 2=\mathrm{P} 1$ then remove $\mathrm{P} 1$ from $\mathrm{L} 2$.

Name the First Polynomial in List (L2), P

Remove every Column where it is not exist in $\mathrm{P}$

//End of Rough set for reducing dimension.

\section{Naïve Bayes Network as classifier}

Predict(Itemset T)

foreach class $\mathrm{C}$ in target values

$$
\mathrm{P}(\mathrm{C})=1
$$


International Journal of Artificial Intelligence \& Applications (IJAIA), Vol.4, No.1, January 2013

for each feature $\mathrm{f}$ in $\mathrm{T}$

$\mathrm{N}(\mathrm{c}, \mathrm{f})=$ The number of Itemsets I in Dataset which I.f=T.f and I.Target $=\mathrm{C}$

$\mathrm{pc}=$ The number of Itemsets I in Dataset I.Target $=\mathrm{C}$

$\mathrm{P}(\mathrm{C})=\mathrm{P}(\mathrm{C}) * \mathrm{p}(\mathrm{c}, \mathrm{f}) / \mathrm{pc}$

Select $\mathrm{C}$ with minimum $\mathrm{P}(\mathrm{C})$

\section{Genetic Algorithm as classifier}

//initial poplulation

Do for START_POP times

Select random ItemSet I from Dataset

Generate gene $\mathrm{G}$ from I add it to population $\mathrm{p}$

// iterating

Do for MAX_ITERATE times

Sort Population by Confidence of Genes()

Do for (CROSS_OVER_RATIO * PopulationSize ) times

Select gp1,gp2 from TOP N\% Population

Cross over gp1,gp2 with position random and generate gc1,gc2

Add gc1,gc2

Do for (MUTATION_RATIO * PopulationSize ) times

Select gpfrom TOP N\% Population

Select position random $P$ from $g p$

Generate random number $\mathrm{r} 0$ or 1

If $r=1$ then

GP $[\mathrm{p}]=-1 / /$ don care it

ELSE

GP $[\mathrm{p}]=$ random Category

If population > MAX_POPULATION

Remove Exceed Popultion

SELECT TOP N\% population as rules

Remove rule which has Confidence $<$ MinConfidence

\section{REFERENCES}

[1] L. Zhou, K.K. Lai \& L. Yu, (2010) "Least squares support vector machines ensemble models for credit scoring", Expert Systems with Applications, Vol. 37, No. 1,pp 127-133.

[2] A. F.Atiya, (2001)"Bankruptcy prediction for credit risk using neural networks: a survey and new results",IEEE Transactions on Neural Networks,Vol. 12, No. 4,pp929-935.

[3] G.P. Zhang, M.Y. Hu, B.E. Patuwo, \& D.C. Indro, (1999) "Artificial neural networks in bankruptcy prediction: General framework and cross-validation analysis", European Journal of Operational Research,Vol. 116, No. 1,pp16-32.

[4] R. A. Johnson \& D.W. Wichern, (2007) Applied multivariate statistical analysis (5th ed.), PrenticeHall, Upper Saddle River, NJ.

[5] N. C. Hsieh, (2005) "Hybrid mining approach in the design of credit scoring models", Expert Systems with Applications,Vol. 28, No. 4,pp655-665.

[6] Sh.-T. Luo, B.-W.Cheng \& Ch.-H. Hsieh, (2009)"Prediction model building with clustering-launched classification and support vector machines in credit scoring", Expert Systems with Applications, Vol. 36, No. 4,pp7562-7566.

[7] G. Karels\& A. Prakash, (1987) "Multivariate normality and forecasting of business bankruptcy", Journal of Business Finance Accounting, Vol. 14, No. 4,pp573-593. 
International Journal of Artificial Intelligence \& Applications (IJAIA), Vol.4, No.1, January 2013

[8] A. K. Reichert, C. C. Cho \& G. M. Wagner, (1983) "An examination of the conceptual issues involved in developing credit-scoring models", Journal of Business and Economic Statistics, Vol. $1, \mathrm{pp} 101-114$.

[9] D. West, (2000) "Neural network credit scoring models", Computers and Operations Research,Vol. 7,pp1131-1152.

[10] W. R. Dillon \& M. Goldstein (1984), Multivariate analysis methods and applications, New York, NY: Wiley.

[11] L. C. Thomas, (2000) "A survey of credit and behavioural scoring: Forecasting financial risks of lending to customers", International Journal of Forecasting, Vol. 16,pp149-172.

[12] V. S. Desai, J. N. Crook \& G. A. Overstreet, (1996) "A comparison of neural networks and linear scoring models in the credit union environment”, European Journal of Operational Research, Vol. 95,pp24-37.

[13] K.Y. Tam \& M.Y. Kiang, (1992) "Managerial applications of neural networks: The case of bank failure predictions", Management Science,Vol. 38 No. 7, pp 926-947.

[14] M.C. Chen \& S.H. Huang, (2003) "Credit scoring and rejected instances reassigning through evolutionary computation techniques", Expert Systems with Applications, Vol. 24,pp433-441.

[15] Z., Huang, H.C. Chen, C.J. Hsu, W.H. Chen \& S.S. Wu, (2004)“Credit rating analysis with support vector machines and neural networks: a market comparative study”, Decision Support Systems, Vol. 37,pp543-558.

[16] K. K. Lai, L. Yu, L.G. Zhou \& S.Y. Wang, (2006) "Credit risk evaluation with least square support vector machine", Lecture Notes in Artificial Intelligence, Vol. 4062, pp 490-495.

[17] K. B. Schebesch\& R. Stecking, (2005) "Support vector machines for classifying and describing credit applicants: Detecting typical and critical regions", Journal of the Operational Research Society, Vol. 56,pp1082-1088.

[18] T.Bellotti\& J. Crook, (2009)"Support vector machines for credit scoring and discovery of significant features", Expert Systems with Applications, Vol. 36,pp3302-3308.

[19] T.-S. Lee, C.-C.Chiu, C.-J.Lu \& I.-F.Chen, (2002) "Credit scoring using the hybrid neural discriminant technique”, Expert Systems with Applications, Vol. 23,pp245-254.

[20] R., Malhotra\& D.K. Malhotra, (2002)“Differentiating between good credits and bad credits using neuro-fuzzy systems", European Journal of Operational Research, Vol. 136,pp190-211.

[21] S. Piramuthu, (1999)“Financial credit-risk evaluation with neural and neurofuzzy systems", European Journal of Operational Research,Vol. 112,pp310-321.

[22] C. Gold, A. Holub\& P. Sollich, (2005)“Bayesian approach to feature selection and parameter tuning for support vector machine classifiers", Neural Networks, Vol. 18 No 5-6, pp 693-701.

[23] M.B. Yobas, J. Crook, \& P. Ross, (2000) "Credit scoring using neural and evolutionary techniques", IMA Journal of Mathematics Applied in Business and Industry,Vol. 11, No. 2,pp111-125.

[24] T. S. Lee \& I. F. Chen,(2005) "A two-stage hybrid credit scoring model using artificial neural networks and multivariate adaptive regression splines”,Expert Systems with Applications, Vol. 28,pp743-752.

[25] Y. Ping \& L. Yongheng, (2011) "Neighbourhood rough set and SVM based hybrid credit scoring classifier”,Expert Systems with Applications,Vol. 38,pp11300-11304.

[26] J. Li, L. Wei, G. Li \& W.Xu, (2011)“An evolution strategy-based multiple kernels multi-criteria programming approach: The case of credit decision making”,Decision Support Systems, Vol. 51, pp 292-298.

[27] J. Wang, K. Guo\& Sh. Wang, (2010) "Rough set and Tabu search based feature selection for credit scoring”, Procedia Computer Science,Vol. 1,pp2425-2432.

[28] Ch.-F. Tsai,\&Jh.-W. W, (2008) "Using neural network ensembles for bankruptcy prediction and credit scoring",Expert Systems with Applications, Vol. 34,pp 2639-2649.

[29] L. Nanni\& A. Lumini, (2009) “An experimental comparison of ensemble of classifiers for bankruptcy prediction and credit scoring”,Expert Systems with Applications, Vol. 36,pp3028-3033.

[30] X. Xu, Ch. Zhou, Zh. Wang, (2009) "Credit scoring algorithm based on link analysis ranking with support vector machine”, Expert Systems with Applications, Vol. 36, pp 2625-2632. 
International Journal of Artificial Intelligence \& Applications (IJAIA), Vol.4, No.1, January 2013

[31] J. Lia, L. Wei, G. Li\& W. Xu, (2011) "An evolution strategy-based multiple kernels multi-criteria programming approach: The case of credit decision making",Decision Support Systems, Vol. 51,pp292-298.

[32] G. Wang, J. Hao, J. Ma, H. Jiang, (2011) "A comparative assessment of ensemble learning for credit scoring", Expert Systems with Applications, Vol. 38, pp 223-230.

[33] B-W. Chi, Ch-Ch. Hsu, (2012) "A hybrid approach to integrate genetic algorithm into dual scoring model in enhancing the performance of credit scoring model", Expert Systems with Applications, Vol.39, No. 3, pp 2650-2661.

[34] A. Capotorti, E. Barbanera, (2012) "Credit scoring analysis using a fuzzy probabilistic rough set model", Computational Statistics and Data Analysis,Vol. 56, No. 4, pp 981-994.

[35] Z. Pawlak, (1982)"Rough sets", International Journal of Computer and Information Science,Vol. 11,pp341-356.

[36] Z. Pawlak, (1991) Rough Sets- Theoretical Aspects of Reasoning about Data, Kluwer Academic Publisher, Netherlands.

[37] D. Heckerman, D. Geiger \& D. M. Chickering, (1995) "Learning Bayesian Networks: The Combination of Knowledge and Statistical Data", Machine Learning, Vol. 20,pp197-243.

[38] P. Cheeseman\& J. Stutz, (1996) Bayesian classification (AutoClass): Theory and results, In Advances in knowledge discovery and data mining,Menlo Park, CA: AAAI Press.

[39] M.M. Morales, N.C. Ramírez, J.L.J. Andrade \& R.G. Domínguez, (2004) "Bayes-N: an algorithm for learning Bayesian networks from data using local measures of information gain applied to classification problems". MICAI: Advances in Artificial Intelligence, Lecture Notes in Artificial.

[40] A. Kamrani, W. Rong\& R. Gonzales, (2001) "A genetic algorithm methodology for data mining and intelligent knowledge acquisition”, Computer \& industrial Engineering, Vol. 40 No. 4,pp361-377.

[41] S.P. Deepa, K.G. Srinivasa, K.R. Venugopal\&L.M. Patnaik, (2005) "Dynamic association rule mining using genetic algorithm", Intelligent Data Analysis,Vol. 9, No. 5,pp439-453.

[42] S. Hettich, C. L. Blake, C. J. Merz, (1998). UCI repository of data mining databases, Available from: http://www.ics.uci.edu/ mlearn/MLRepository.html.

[43] H.Jabeen\& A. R. Baig, (2012) "Two layered Genetic Programming for mixed-attribute data classification”,Applied Soft Computing,Vol. 12,pp416-422.

\section{Biographical notes}

Ali Zinal-Hammadani is a professor in Department of Industrial Engineering at Isfahan University of Technology, Isfahan, Iran. His research interests are in the areas of statistics, Quality control, Multivariate statistical analysis, Data mining and reliability modeling. He has numerous publications in international journals and conferences

Ali Shalbafzadeh received his MS degree in software engineering from Isfahan University of Technology, Iran, in 2011. His research interests include data mining and intelligent systems.
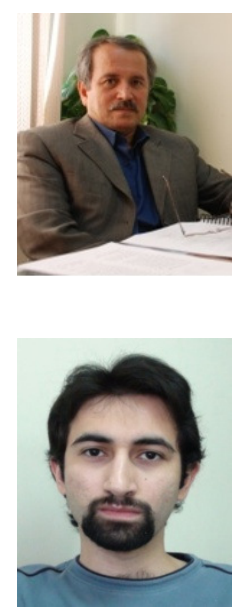
International Journal of Artificial Intelligence \& Applications (IJAIA), Vol.4, No.1, January 2013

TaghiRezvan is a $\mathrm{PhD}$ student in Department of Industrial Engineering at Isfahan University of Technology, Iran from 2009. His research interests are in the areas of data mining, operation research, risk management and intelligent systems. He has published several journal papers and conference papers in the above filed.

AfshinShahlaieMoghadam received his MS degree in industrial engineering from Isfahan University of technology university, Iran. His fields of interests are: financial management, strategic implementation Model especially balanced scorecard, IT project management, Risk Analysis.
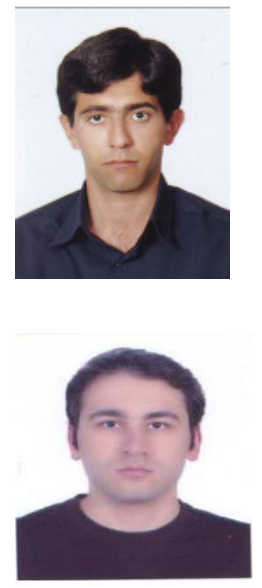